\title{
DESIGN DE MATERIAIS COMO UMA DEMANDA DA CIÊNCIA E TECNOLOGIA PARA A INOVAÇÃO
}

\section{MATERIALS DESIGN AS A DEMAND FROM THE SCIENCE AND TECHNOLOGY TO INNOVATION}

\section{Debora Barauna ${ }^{1}$, Dalton Luiz Razera², Michele Tais D. C. Zamoner ${ }^{2}$, Denise Abatti Kasper Silva ${ }^{3}$}

\section{RESUMO:}

O avanço do conhecimento na ciência e tecnologia dos materiais possibilitou a manipulação e a criação de novos materiais com melhores propriedades em atendimento a aplicações específicas. Dentro desse contexto, o presente artigo visa elucidar o termo design de materiais mediante a promoção da inovação. Foi realizada uma revisão narrativa da literatura e dissertado sobre o tema a partir de períodos históricos de desenvolvimento da sociedade. Os procedimentos de pesquisa utilizados foram pesquisa bibliográfica e análise de conteúdo. Os resultados obtidos mostram o design de materiais como uma abordagem demandada pela engenharia de materiais no final do século 20 , quando o pensamento preditivo foi adotado para a pesquisa, o desenvolvimento e a aplicação de materiais avançados. Já no século 21 o conceito do design de materiais compreendeu os conhecimentos do design e da gestão com o propósito de aumentar a probabilidade de sucesso da difusão da inovação para a sociedade. A colaboração e a criatividade são as apostas emergentes nesse processo.

PALAVRAS-CHAVE: materiais avançados; colaboração; criatividade; processo de design.

\section{ABSTRACT:}

The advancement of knowledge in the materials science and technology made possible the manipulation and creation of new materials with improved properties to specific applications. In this context, the aim of this study was to elucidate the emerging approach of the materials design toward promoting innovation. A narrative literature review on the subject based on historical periods of society development. The research procedures used were bibliographic research and content analysis. The results show the material design as an approach demanded of the materials engineering, born at the end of the 20th century, where the predictive thinking was adopted for the development and application of advanced materials. Already in the 21st century the concept of materials design comprised design and management knowledge in order to increase the probability of success of the diffusion of innovation to society. The collaboration and creativity are emerging bets in this process.

KEYWORDS: advanced material; collaboration; creativity; design process.
Fonte de Financiamento: Coordenação de Aperfeiçoamento de Pessoal de Nivel Superior; Universidade Federal do Paraná, Programa de PósGraduação em Design.

Conflito de Interesse: Declara não haver.

Ética em Pesquisa: Declara não haver necessidade.

Submetido em: 04/09/2017 Aceito em: 09/01/2021 


\section{INTRODUÇÃO}

Nas áreas técnicas e científicas o que se observava, até pouco tempo atrás, era uma segregação do conhecimento em disciplinas e a formação de especialistas. No entanto, com o alargamento da complexidade das relações entre a sociedade e o meio ambiente, ações interdisciplinares e transdisciplinares em processos de promoção da inovação tornaram-se cada vez mais proeminentes. Paralelo a isso, houve o desenvolvimento acelerado da sociedade com o avanço das tecnologias de comunicação, que promoveram maior acesso à informação e a disseminação do conhecimento. Assim, em favor da compreensão dos problemas complexos surgiram novas abordagens do design e da gestão associadas ao pensamento sistêmico e à colaboração, que diminuíram barreiras de integração de processos e facilitaram a interação entre partes interessadas (stakeholders).

Entretanto, no campo da ciência e tecnologia dos materiais, a integração e a interação com demais áreas do conhecimento, interessadas no processo de inovação em materiais, tecnologias e produtos, podem ser melhoradas, dinamizadas e humanizadas, principalmente, considerando a realidade brasileira e de outros países subdesenvolvidos. Diversos fatores de insucesso são evidenciados nas invenções desse campo, principalmente, relacionados com o longo tempo de duração que as invenções demandam para obterem resultados. Na perspectiva de Mcdoweel e Story (1998) e também de Olson (2001) que corrobora com essa visão: até o século 20 os materiais eram descobertos por acaso, após o achado de um material, propriedades técnicas eram determinadas e só então formas de uso ou aplicações em produtos eram exploradas. Porém, diante do atual ritmo de desenvolvimento da sociedade, tais autores previam que esses processos tornariam-se demasiadamente trabalhosos, intensivos, dispendiosos e poucos ousados para enfrentar os desafios da economia do século 21 .

Neste século, às fases de pesquisa e desenvolvimento (P\&D) de um material, tecnologia e produto passaram a incorporado o processo de inovação. Esta é uma estratégia que vem sendo chamada de pesquisa, desenvolvimento e inovação (PD\&I) e é nesse universo que a ideia do design de materiais reside.

A estratégia PD\&I e o design de materiais são ambas abordagens que surgiram com a intenção de aumentar a probabilidade de sucesso de uma invenção. 0 fato é que a partir do advento dos materiais avançados, novos processos de concepção foram lançados e, como isso, os materiais ganharam nova significância na promoção da inovação. Porém, a questão levantada neste estudo é como o termo design de materiais compreende a relação entre as áreas da ciência e tecnologia dos materiais e do design para a promoção da inovação. Desta forma, o presente artigo visou elucidar o termo design de materiais diante da promoção da inovação. Foi realizada uma revisão narrativa da literatura sobre o tema, norteada pela evolução histórica de desenvolvimento da sociedade. Uma vez que, a história mostra que os avanços na ciência e tecnologias dos materiais e a evolução da sociedade estão mutuamente conectados.

A seguir o método de pesquisa empregado no estudo é fundamentado, os resultados obtidos são descritos e a conclusão é apresentada. Mostra-se que, embora, a abordagem do design de materiais possa parecer um termo oriundo da área do design, trabalhos publicados (MCDOWELL, 1998; OLSON, 2001; DOBRZANSKI, 2006; MESSER et al. 2007; CGEE, 2010; BAYKARA, 2015) demonstram o surgimento da prática por meio da engenharia de materiais ainda no século 20. Entretanto, no século 21 a visão sobre a abordagem é ampliada, passando a envolver os conhecimentos da área do design e da gestão para a promoção da inovação. Por fim, o design de materiais e a estratégia PD\&I são abordagens convergentes na Sociedade Contemporânea, em que a colaboração e a criatividade destacam-se diante de um cenário voltado para uma Economia Criativa. 


\title{
MÉTODO DE PESQUISA
}

O método de revisão narrativa da literatura (RNL), empregado no estudo, refere-se a uma pesquisa de mapeamento com levantamento bibliográfico amplo e aberto de trabalhos publicados (VOSGERAU e ROMANOWSKI, 2014). Nesse contexto, foram utilizados procedimentos de pesquisa bibliográfica para a coleta de dados e análise de conteúdo, do tipo temática, para a seleção e interpretação das informações obtidas. Por meio da análise de conteúdo foi possível gerar uma sintetize e contextualização do tema proposto. Foram realizadas relações de conteúdos de produções relevantes encontradas entre o final século 20 e início do século 21. Por fim, foram apontadas perspectivas de caminhos para novas pesquisas, tal como orientam os autores indicados na conceituação do método de RNL.

\section{DESIGN DE MATERIAIS}

Ao longo do tempo o uso dos materiais tornou-se parte integrante da cultura humana como a substância da ação, o que conferiu a este estudo tratar os materiais de modo indissociável da técnica (tecnologia) e do uso (produto). Os materiais são empregados em produtos de uso cotidiano ou para fins específicos por meio do conjunto de saberes empregados, a tecnologia. o Centro de Gestão e Estudos Estratégicos (CGEE) afirma que:

\begin{abstract}
Um dos aspectos característicos do progresso da civilização humana foi sua dependência no desenvolvimento e na aplicação de novos materiais, o que os levou a servir como marco temporal de diferentes etapas da evolução das sociedades, desde os primórdios de nossa história. Nas últimas décadas [...] a relevância dos materiais no desenvolvimento tecnológico tem aumentado significativamente, tornando-os, em muitos casos, fatores determinantes para a introdução de novas tecnologias e agentes fundamentais do processo de inovação. (CGEE, 2010, p.8)
\end{abstract}

A divisão dos períodos históricos de desenvolvimento da sociedade mostra que durante a Era Moderna (entre os séculos 18 e 19) diversas revoluções tecnológicas ocorreram e alavancaram o desenvolvimento de novos processos e produtos. A partir desses avanços houve um rápido crescimento na experimentação e no desenvolvimento de novos materiais (NAVARRO, 2006). Desde então, o conhecimento gerado pela ciência e tecnologia dos materiais vem sendo utilizado no desenvolvimento de produtos novos ou na melhoria desses (BELL, 2011).

Foi, justamente, na Era Moderna que o design e a ciência e tecnologia dos materiais estabeleceram-se como campos do conhecimento. 0 design foi alavancado pela Revolução Industrial no final do século 19 com o surgimento das máquinas que gerou a produção em massa de produtos e substituiu o processo artesanal (MORAES, 1999). Já, no início de 1900, o domínio da ciência e tecnologia dos materiais resultou da compreensão atomista que determinou as ciências da física e da química, possibilitando a relação entre as propriedades de um material e de suas microestruturas (BELL, 2011). De igual modo ao design, os conhecimentos científicos e tecnológicos começaram a interagir mediante aos estímulos da expansão comercial e do crescimento acelerado da cultura urbana - a partir da criação de novas tecnologias, ferramentas e máquinas, os cientistas foram capazes de obter maior compreensão dos fenômenos (DOBRZANSKI, 2006). Segundo Padilha (2000) tal relação não se devia apenas a uma influência unidirecional da ciência e tecnologia, havia uma sinergia entre essas atividades para uma atuação conjunta, como uma engenharia.

Na ciência sabe-se como ocorrem os fenômenos e os seus motivos, na tecnologia sabe-se fazer com base científica e precisão e na engenharia deve-se saber como conceber e projetar, além 
de montar, operar, executar e otimizar, com base científica e tecnológica (ZAKON, NASCIMENTO e SZANJBERG, 2003). Assim, na década de 1950 a ciência e tecnologia dos materiais foram trazidas em conjunto pela física e química como uma única disciplina, a engenharia de materiais (OLSON, 2001; IEEE-UFABC, 2015). Essa formou especialistas na área da ciência e tecnologia dos (i) metais, (ii) materiais cerâmicos, (iii) polímeros e (iv) compósitos para aplicações estruturais e eletrônicas, que colaborou para a busca de princípios unificadores na criação de materiais por essas respectivas classes (OLSON, 2001), denominados de materiais modernos ou tradicionais. Navarro (2006) afirma que a partir da unificação das classes de materiais houve uma revolução nos conhecimentos e desenvolvimentos de novos materiais diante de modificações nas formulações e estruturas de materiais tradicionais.

\begin{abstract}
Em meados do século 20 iniciou-se uma nova era de materiais. Essa expressão mexeu com o mundo antigo e próspero da matéria. Cada sociedade e período retrata sua imagem dominante em um tipo particular de material [...], mas a sociedade contemporânea parece ter realmente virado a mesa e provocado um frenesi de materiais considerados "novos". [...] o mundo novo e infinito dos materiais que selecionamos, escolhemos e combinamos para produzir outros, de cada vez mais alto desempenho. (KULA e TERNAUX, 2012, p.315)
\end{abstract}

Na contemporaneidade a engenharia de materiais estuda o efeito da estrutura da matéria em nível atômico, ou seja, por várias escalas de elétrons (cristalino, micro e macro) para que novos materiais com propriedades funcionais e desejadas possam ser criados (PADILHA, 2000; DOBRZANSKI, 2006; BELL, 2011). Esses são definidos como materiais avançados, mas também conhecidos como materiais high-tech, materiais futuros, materiais de alta performance, materiais de alto valor, materiais melhorados e materiais finos (MEDINA e NAVEIRO, 1998; BEL, 2011; BAYKARA, 2015). De modo geral, todos esses termos sugerem que os materiais avançados possuem melhores propriedades, que os materiais tradicionais, em atendimento a um setor ou uma demanda em particular (XIONG, SUN e JIANG, 2008). Esses são obtidos por meio de:

Processos de síntese da matéria prima, com controle das características estruturais do material, e foco em desempenho sistêmico do produto acabado[...]. (CGEE, 2010, p.7)

Enfim, a manipulação desses processos permitiu que estudos da engenharia de materiais passassem a ser chamadas design de materiais ou materiais projetados.

Ao contrário dos materiais descobertos até a Era Moderna, as mudanças ocorridas na contemporaneidade aconteceram em intervalos de tempo tão dinâmicos, que para Padilha (2000) esses refletem em verdadeiras revoluções. Nesse contexto, o autor classificou e exemplificou em quatro níveis o grau de conhecimento científico e tecnológico empregado nos materiais até então, tais como:

- Nível 1 - Materiais naturais (madeira, couro, diamante, cobre, ligas ferrosas e borracha).

- Nível 2 - Materiais desenvolvidos empiricamente (bronze, aço comum, ferro fundido, cerâmicas sílico-aluminosas, vidro, cimento e concreto).

- Nível 3 - Materiais desenvolvidos com conhecimentos científicos em relação aos seus descobrimentos e à interpretação das qualidades de suas propriedades (ligas mais 
antigas de alumínio, de titânio e de magnésio, metal duro, aços inoxidáveis, aços microligados, termoplásticos, termorígidos, elastômeros e ferritas).

- Nível 4 - Materiais novos ou aperfeiçoados, projetados com base em conhecimentos científicos, quanto à previsão quantitativa de suas propriedades (semicondutores, materiais para reatores nucleares, aços de alta resistência mecânica, compósitos reforçados com fibras, ligas com memória de forma e vidros metálicos).

Desta forma, os materiais projetados são derivados do design de materiais, uma abordagem em que métodos científicos e de design são utilizados em sistemas de engenharia para adequar estruturas materiais e modos de processamento na obtenção de aplicações específicas e inovadoras (MESSER et al., 2007). Com essa perspectiva, Baykara (2015) afirma que as relações entre (i) propriedades, (ii) estrutura, (iii) processo e (iv) performance, propostas no esquema clássico em pirâmide da engenharia de materiais, não são mais válidas para o recémformado sistema complexo e dinâmico do design de materiais. Uma nova proposição de relações na pirâmide é sugerida pelo autor entre (i) materiais nano-estruturados; (ii) design, modelagem e simulação; (iii) adaptação e (iv) funções e multifunções. Por fim, o autor considera que, na passagem para o século 21 , com o rápido avanço das nanociências e nanotecnologias, junto ao desenvolvimento de instrumentos analíticos altamente capazes e eficazes para a realização de testes e caracterização de micro e nanoestruturas (por exemplo, microscópios eletrônicos de resolução ultra elevada) conduziram a engenharia de materiais para uma nova era de relações. Estas voltadas para o propósito do design de materiais como um caminho sistêmico para a promoção da inovação.

\section{PROMOÇÃO DA INOVAÇÃO}

Na ciência e tecnologia dos materiais, tradicionalmente, o processo de inovação tem se caracterizado por um longo período de gestação entre a invenção, a primeira aplicação comercial e a sua utilização generalizada (MAINE, PROBERT e ASHBY, 2005; XXVI PRÊMIO JOVEM CIENTISTA, 2012). Historicamente, entre 1970 a 1980 o tempo de difusão da inovação em materiais, considerando a descoberta científica até a aplicação comercial, levava em torno de 10 a 15 anos para ocorrer, já nos anos de 1990 o tempo passou a ser de 5 a 7 anos (MEDINA e NAVEIRO, 1998). Na realidade brasileira um novo material demora em média de 5 a 10 anos para entrar no mercado (NASSOUR, 2008).

No Brasil, concorda-se com a visão de Nassour (2008) em que ele afirma que esse período se refere ao tempo necessário, inicialmente, para se obter o fomento de apoio para a realização da pesquisa, que ainda ocorre em laboratórios de universidades e institutos de pesquisas do país e que depois se desdobra nas seguintes questões: primeiro o material deve ser sintetizado; após ele sofre uma série de testes e experimentos; em seguida são realizados estudos de processos necessários para absorção do material pelo mercado - a isto vincula-se à capacitação de recursos humanos, à elaboração de protótipos das aplicações e ao desenvolvimento de pesquisas de mercado. Por fim, até chegar ao consumidor final, o autor poderá que a transferência de tecnologia pode ainda barrar em outras questões, tal como, não encontrar um fabricante interessado na produção, na burocracia técnica envolvida e na avaliação do seu impacto ambiental.

0 fato é que ao longo desse caminho muitos aspectos negativos podem obstruir a promoção da inovação (XIONG, SUN e JIANG, 2008; KINDLEIN e GUANABARA, 2006; MAINE, PROBERT e ASHBY 2005; MEDINA e NAVEIRO, 1998). Em primeiro lugar está o fator custo do processo, relacionado, diretamente, com o longo tempo de maturação da invenção bem como com os preços dos insumos e equipamentos, dos testes de aplicação e da padronização da produção até à certificação final do produto e/ou processo (MEDINA e NAVEIRO, 1998). Outro fator negativo para a inovação em materiais avançados está na necessidade da adoção de novas 
tecnologias de fabricação. Isso envolve tanto mudanças de processos de produção como de cultura organizacional, além da capacitação de recursos humanos para a fabricação em escala industrial. Normalmente, desenvolver materiais avançados custa mais caro que aplicar materiais tradicionais com tecnologias já prontas, no entanto, a produção seriada pode contribuir para redução do custo, mas alguns materiais avançados encontram dificuldades neste processo de escalonamento da produção (XXVI PRÊMIO JOVEM CIENTISTA, 2012).

Materiais avançados e alta tecnologia contemplam um ao outro, deste modo, os elevados riscos de investimento na criação ou modificação de processos fazem com que os fabricantes hesitem em aplicar novos materiais em produtos. Por outro lado:

o grau de conhecimento científico empregado no desenvolvimento de um material avançado tem efeito determinante no seu preço e a capacidade de produzi-lo é uma medida do grau de desenvolvimento tecnológico (e independência) de uma nação. (PADILHA, 2000, p.23)

Chen (2012) corrobora com essa visão ao ponderar que investimentos em novos materiais e novas tecnologias são fatores-chave para o crescimento em ambientes de rápidas mudanças e para a rentabilidade das empresas. Com isso, a decisão de inovar das organizações exige altos investimentos e uma atitude para agir na incerteza, pois o retorno não é garantido, mas a decisão de não inovar também é arriscada, podendo até determinar a extinção de uma empresa do mercado (BAXTER, 2011).

Entretanto, pouco tem se discutido sobre a difusão da inovação em materiais avançados (CHEN, 2012). A difusão da inovação de materiais avançados no mercado é um desafio atrelado ao gerenciamento da tecnologia (MAINE, PROBERT e ASHBY, 2005). Assim, o pensamento sistêmico tem se destacado na concepção e aplicação dos materiais avançados. A integração de fases e a colaboração entre as partes envolvidas exige uma visão holística sobre o processo, permitindo a elaboração de um projeto com uma perspectiva inicial do todo do processo, isto, tanto na aplicação de materiais e tecnologias conhecidas pela indústria como no desenvolvimento de novos.

\section{PENSAMENTO SISTÊMICO}

Baseado em Maine (2000 apud Maine, Probert e Ashby, 2005) a evidência empírica mostra que a conectividade, junto à tendência para a pesquisa colaborativa, tem sido uma alternativa promissora de acesso ao capital. Experiências no sentido dessa integração pressupõem um 'consorciamento' entre as partes interessadas e rompem barreiras das fases de pesquisa, desenvolvimento e inovação dos materiais avançados, com especialistas trabalhando juntos em um projeto concebido por todos desde o início (MEDINA e NAVEIRO, 1998). Para os autores, os resultados dessa integração e interação revelam-se em: maior competitividade para o produtor; satisfação do usuário; minimização de tempo, trabalho e recursos financeiros despendidos; redução de impactos ambientais; maximização de desempenho e segurança e competências desenvolvidas nos envolvidos com o processo - a parceria entre especialistas torna o próprio processo uma forma de aprendizado, gerando e transferindo conhecimentos para toda a equipe executora.

Em particular, tal atuação sistêmica tem refletido em uma redução significativa de tempo para a difusão da inovação. A exemplo disso, destaca-se o caso de inovação dos multichips em indústrias eletrônicas: 
[...] as firmas que seguiram o processo tradicional: pesquisa básica (ciência dos materiais), pesquisa aplicada (busca de usos), desenvolvimento experimental (protótipo), testes em pequena escala até a introdução do novo material na linha de produção, levaram 6,5 anos, envolvendo 800 pessoas. Já pelo enfoque sistêmico outras firmas obtiveram o mesmo resultado em 4 anos com equipes multidisciplinares de 300 pessoas entre: cientistas, pesquisadores, técnicos e engenheiros, trabalhando juntos. (MEDINA e NAVEIRO, 1998, p.30)

Por fim, Olson (2001) reconhece, em sua publicação marco (Beyond Discovery: Design for a New Material World) os avanços em curso na Teoria Geral dos Sistemas apontados por Cyril Stanley Smith, que defendia uma visão sistêmica dos materiais como um caminho mais frutífero para o futuro de uma ciência útil para a complexidade natural. Contudo, o autor pondera que um padrão consistente na evolução da ciência e tecnologias dos materiais, ao longo de quatro décadas (1960 a 2000) moveu-se em direção à boa ciência ao invés de bons materiais. Para Olson (2001) um modo de oportunizar materiais melhores e de reduzir o tempo e o custo de desenvolvimento desses é explorar, extrinsecamente, maior previsibilidade dos sistemas de concepção.

\section{PENSAMENTO PREDITIVO}

As dificuldades para a inovação na engenharia de materiais são intrínsecas aos sistemas baseados em descobertos, em vez de projetados: um bom design requer muito menos desenvolvimento (OLSON, 2001). 0 autor faz alusão à demanda de conhecimentos do design na engenharia de materiais e aponta para o futuro do design de materiais computacional. É indicado a integração da engenharia de materiais a modelagem preditiva para simulação e desenvolvimento integral do material ao produto.

Já, com essa perspectiva, em 1985 o Steel Research Group (SRG) desenvolveu uma metodologia de design de materiais computacional para o ensino. Com isso, a Universidade Northwestern em 1989 implantou o primeiro curso de graduação intitulado Design de Materiais. 0 curso apresentava uma série de sessões computacionais de ensino baseado na criação de bancos de dados genoma de materiais fundamentais (Genomic Material Design). Isto integrado à abordagem do desenho gráfico paramétrico. Os estudantes do curso da graduação apoiavam ainda os projetos dos alunos do Programa de Doutorado Interdisciplinar em Ciência Preditiva e Design de Engenharia (Predictive Science and Engineering Design, DESP) (OLSON, 2013).

Já no século 21 surgiu também o software Design de Materiais Baseado em Computador (Computer Aided Material Design,CAMD) voltado para a concepção de materiais sustentáveis. 0 $C A M D$ engloba o desenvolvimento por completo dos materiais avançados, com propriedades desejadas, além de aspectos ambientais e de consumo reduzido de energia (DOBRZANSKI, 2006).

Para o futuro, segundo o National Research Council (2004) a aposta é, cada vez mais, um desenvolvimento totalmente integrado entre práticas de design, desenvolvimento de materiais e fabricação, em um ambiente computacional contínuo, evoluindo de um processo de atuação interdisciplinar para uma atuação transdisciplinar (Figura 1). 
Figura 1. Modelo de transição para 0 desenvolvimento acelerado dos materiais no futuro

Fonte:

Baseado em National Research Council (2004).

\section{Abordagem atual}

para a transição de materiais

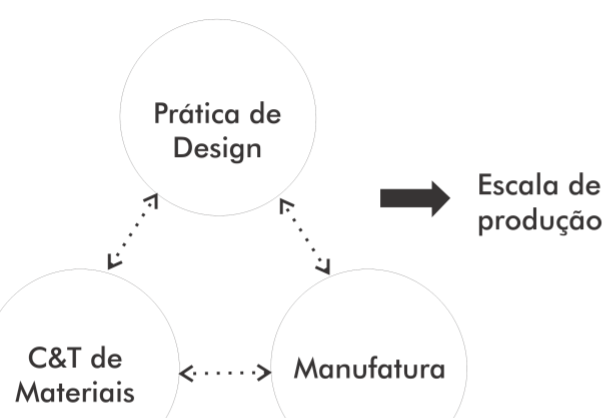

Equipes integradas guiadas por um processo de desenvolvimento disciplinado

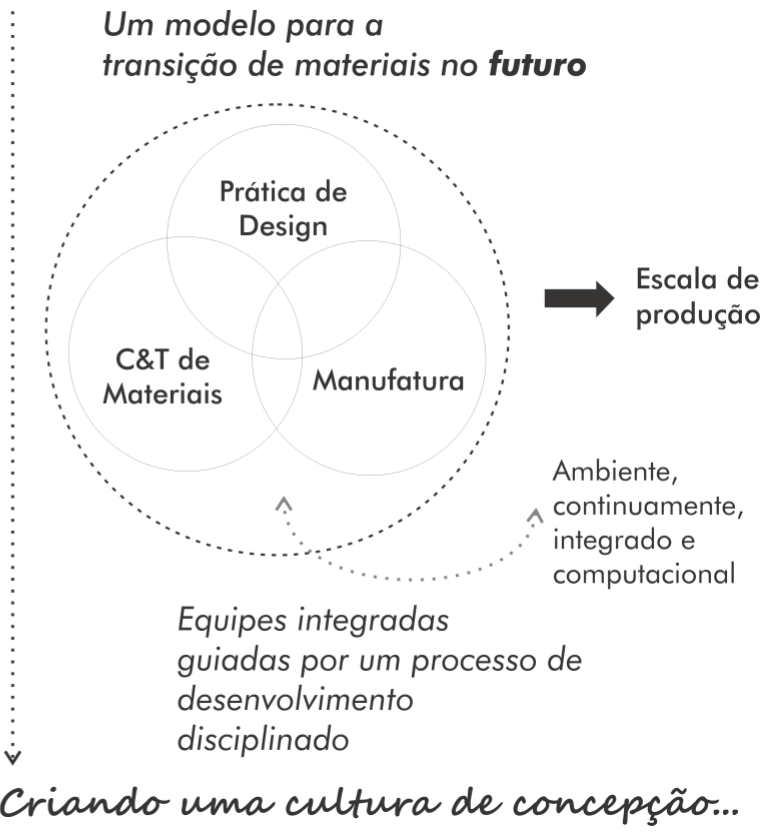

Os sistemas preditivos vão além de um sistema de tecnologia por acidente, eles buscam oferecer benefícios sociais substanciais, bem como uma nova justificativa para o investimento científico e tecnológico (OLSON, 2013). Assim, para além do design de materiais computacionais, ainda na primeira década do século 21 surgiram diversos métodos ou modelos de processos que integraram os conhecimentos do design e da gestão da inovação ao desenvolvimento de novos materiais e tecnologias. Embora a origem desses métodos é variada, todos projetam e compartilham o mesmo fim de aumentar a probabilidade de sucesso comercial do material. Alguns exemplos desses métodos são:

- Accelerated Insertion of Materials (AIM), criado pela Defence Advanced Research Projects Agency dos Estados Unidos, é um método para a inserção acelerada de materiais, com o objetivo de utilizar recursos de modo mais eficiente, por meio do processo de design, e estabelecer as propriedades dos materiais necessárias nas fases iniciais do desenvolvimento dos materiais (THONG, JACKSON e ANDERSON, 2009).

- Investment Methodology for Materials (IMM) é outro método de investimento para materiais que avalia a viabilidade técnica e econômica da inovação em materiais, bem como a probabilidade de capturar valor pela adoção da inovação. 0 método é divido em três segmentos: viabilidade, avaliação de mercado e valor. No IMM um material é viável em uma aplicação quando há equilíbrio entre os seus atributos técnicos e econômicos. O IMM contribui para a identificação do mercado promissor e previsão do volume de produção, também, propõe a integração e simultaneidade do processo (MAINE, PROBERT e ASHBY, 2005).

- Pre-commercial Technology-Push Design Strategy é um modelo de processo desenvolvido pela CRC Wood Innovations a fim de facilitar a colaboração entre a ciência, a engenharia e o design no desenvolvimento de novos materiais. No modelo, requisitos para a aplicação ou para o produto são descritos como critérios de desempenho das propriedades do material e especificados como metas de pesquisa para a ciência e engenharia. Em uma segunda etapa é estabelecido o quadro da colaboração na condução da pesquisa, de modo simultâneo, entre as áreas do design, da ciência e da engenharia. 0 modelo incita o design a trabalhar com a concepção de novos materiais ao invés de só novos produtos (THONG, JACKSON e ANDERSON, 2009) 
- Acoplamento das abordagens de technology push (impulso tecnológico) e market pull (demanda de mercado) é sugerida por Baykara (2015) como um mecanismo com potencial elevado para encontrar novos modelos de negócios e resultados de colaboração na comercialização dos materiais avançados. 0 modelo de acoplamento de abordagens proposto pelo autor, considera uma cadeia de valor típica para materiais avançados. 0 design e a estratégia de PD\&I são destacados, conjuntamente, nas etapas de matéria prima e processamento da proposta de cadeia de valor dos materiais avançados sugerida pelo autor.

A necessidade de construção desses métodos e modelos, bem como a visão de futuro do National Research Council (2004) com a integração entre práticas de design, desenvolvimento de materiais e fabricação deve-se, então, ao aumento gradativo da complexidade dos sistemas, decorrente, em parte, tanto do novo padrão de acumulação do conhecimento como da nova perspectiva de consciência humana que se estabeleceu na contemporaneidade. Segundo Dobrzanski (2006) o potencial de inovação associado ao design de materiais é impulsionado pelo amplo conhecimento sobre os materiais existentes, mas também pelos avanços nas tecnologias de informação e comunicação (TICs) que dinamizaram as relações sociais e criaram novas formas de cultura, como a cibercultura ou inteligência coletiva. Na Sociedade Contemporânea, os consumidores estão mais conectados, exigentes e atentos às inovações que contribuem significativamente para a sociedade, seus modos de vida e o meio ambiente. Assim, Thong, Jackson e Anderson (2009) afirmam que a integração da engenharia de materiais com o design tem resultado em acelerados prazos de entrega da inovação, menor risco de desenvolvimento e melhor aceitação de materiais, tecnologias e produtos pelo mercado. Olson (2001) já apontava que o valor de um produto resulta apenas em parte do atendimento de questões técnicos e muito mais da interação com o consumidor, o valor humano traz diversidade criativa para o processo e potencial de avanço social.

\section{PENSAMENTO CRIATIVO}

No século 21, a inovação no design de materiais relaciona-se tanto com a recente influência do usuário nos processos de tomada de decisão e escolhas de materiais quanto com o desempenho do produto acabado. 0 desempenho em materiais avançados, de acordo com Medina e Naveiro (1998) referem-se, basicamente, as propriedades finais dos materiais, resultantes das suas caracterizações físicas, mecânicas, térmicas, elétricas, ópticas, magnéticas, biológicas e químicas para uma aplicação fim. Dobrzanski (2006) complementa que várias dessas propriedades podem ser obtidas em materiais compósitos, no entanto, tais propriedades dependem da estrutura, da composição química e das condições de uso, como por exemplo: cargas cíclicas em uso de alta ou baixa temperatura; presença de meios que provocam a corrosão geral; fissuras resultantes de corrosão sob tensão etc. Já o design contemporâneo, bem diferente daquele nascido a partir da Revolução Industrial, preocupa-se mais com a criação de sentido, com a produção de valor para a sociedade e o meio ambiente. Assim, seus cuidados de projeto vão além de funções técnicas e de uso, visam criar funções simbólicas aos produtos, conforme explica-se por Voos (2012) a seguir:

- Funções simbólicas - caracterizam-se pela atribuição de significados aos produtos, adquiridos por experiências passadas dos indivíduos e relacionados com os sentidos, as percepções, as emoções e as associações à época e cultura, por exemplo.

- Funções de uso - compõem a configuração formal e espacial do produto (tamanho, peso, pegas e proporções) e os modos de uso necessários em relação à dimensão física corporal do usuário, aos movimentos pertinentes para o desempenho da tarefa e à manipulação do produto. Essas questões são relativas aos estudos da ergonomia. 
- Funções técnicas - decorrem do atendimento das duas outras funções, simbólica e de uso, que no processo de design são traduzidas em atributos de projetos. Esses visam orientar a tomada de decisão de escolhas projetuais, que por vez influenciarão as escolhas dos recursos que serão necessários para o desenvolvimento do projeto, como por exemplo, o uso de tecnologias específicas, matérias primas e processos de fabricação. Esses recursos podem já existir ou a concepção desses poderá ser requerida.

Contudo, o que se observa ainda em trabalhos publicados da engenharia de materiais é que o uso do termo design de materiais, muitas vezes, não considera as funções do design em sua totalidade. As funções técnicas na engenharia de materiais não cobrem as outras duas funções enfatizadas pelo design, pelo menos não as funções simbólicas. Olson (2001) já orientava que, com o estímulo da concorrência global, os métodos para o design de materiais deviam moverse da eficácia para alta eficiência. A ideia era que, com os ciclos de feedbacks do processo de design, em que se diverge e converge sobre diferentes visões, o valor humano fosse trazido para dentro do processo de design de materiais.

Segundo Baykara (2015) no início do século 21 uma nova Era para o design de materiais emergiu influenciada pela dinâmica do mercado e intensa competição, nessa nova Era de concepção avançada, o design de materiais tem exigido atributos como: inovação e criatividade; rápida implantação e comercialização das aplicações e ampla colaboração em PD\&I. Para o autor, a inovação e a criatividade em tecnologias de materiais avançados estão se tornando os principais pilares para quase todas as organizações, em que, a colaboração em rede é um novo paradigma para os materiais avançados, já que a maioria das ideias e aplicações inovadoras tem envolvido extensa colaboração entre universidades e empresas para se obter novas soluções baseadas na ciência e prática. 0 autor ainda complementa que essa nova Era cada vez mais se dirige para o desenvolvimento de materiais e produtos com alto valor agregado; sendo esses:

- multisetoriais (energia, transportes, médica, esportes, embalagens, aeroespacial, engenharia civil e outros);

- multifuncionais (mecânica, física, química, elétrica e outros);

- entre múltiplas disciplinas (física, química, matemática aplicada, biologia, mecânica, elétrica, eletrônica, engenharia, design, administração, sociologia, psicologia etc.)

- entre práticas de distintas tecnologias (biociências, nano-eletrônicas, fotônicas, mecatrônicas entre outros).

Um material é um sistema complexo por si só (MCDOWELL, 1998). No entanto, é na aplicação de novos materiais que se constitui uma alteração na Era e não apenas na existência de uma tecnologia de materiais avançados (VERGHESE, 2008). Assim, o conhecimento do design de materiais deve incluir um novo pensamento sobre o material, para que esse responda tanto aos avanços dos processos tecnológicos como às exigências sociais por novas tecnologias. $\mathrm{A}$ proposta é que, na concepção de novos materiais pelo design, se tenha a inovação como a mudança que se sucede pela abordagem centrada no ser humano, destacando a interação material-produto-usuário (ver Barauna et al., 2017). Os materiais avançados não constituem uma mudança por si só. É a aplicação desses dentro de um contexto, que questões de concepção de uma nova Era do Design configuram-se (VERGHESE, 2008).

Desde a passagem para o século 21 a ideia de melhor concepção do uso da matéria vem sendo reportada na ciência e nas práticas. Particularmente, na engenharia de materiais houve uma evolução da Era das Descobertas para a Era do Design, como denominou Olson (2001) que caracterizou esse período também como o desenho de um novo mundo material. Já Verghese 
(2008 e 2009) chamou isso de ideias perigosas do design e design às margens referindo-se à inovação e à necessidade de ultrapassarmos as margens das fronteiras do conhecimento.

Em suma, nessa vertente de concepção humana dos materiais avançados, o designer tem se destacado como o agente pensante, dirigindo a mudança nos processos de PD\&I, o que exige propor novas concepções de design voltadas para pensar materiais, tecnologias e produtos, simultaneamente (ver Barauna e Razera, 2019). Compreende-se que, embora, no passado, materiais, tecnologias e produtos tenham-se configurado, separadamente, dentro de áreas específicas de atuação, suas histórias mostram que esses campos de conhecimento foram dependentes entre si. Padilha (2000) apontou que sempre houve uma sinergia entre a ciência e a tecnologia. Todavia, na contemporaneidade o valor humano tem ampliado essa relação ao design, bem como à gestão da inovação.

\section{CONCLUSÃO}

Os métodos da ciência e tecnologia dos materiais, normalmente, baseados em erros e acertos por experimentos, com estudos dispendiosos e incertos, em que, muitas vezes, os pesquisadores desconhecem as possibilidades de aplicação e aceitação dos seus achados, voltam-se agora para a concepção do design de materiais.

Tal tendência é tanto impulsionada pelos avanços no conhecimento da ciência e tecnologia dos materiais, que hoje permitem manipular novos materiais ou materiais avançados, como pelo maior acesso à informação; pela expansão do livre comércio; pelas pressões ambientais e sociais existentes e pela inserção do usuário e demais partes interessadas nos processos de concepção, que se estruturam em redes colaborativas e inovativas.

Assim, ainda no século 20, o termo design de materiais surgiu como uma demanda da engenharia de materiais para a promoção da inovação, sendo associado ao processo de design, primeiro, pelo uso do pensamento sistêmico e preditivo e, mais tarde, pelo valor humano e sua criatividade.

No século 21, de modo emergente, o processo de design tem se destacado na cadeia de valor dos materiais avançados, junto à estratégia PD\&I, como um agente determinante na identificação de reais demandas da sociedade e na criação de valor, quem resultam, em parte, da colaboração entre stakeholders e da criatividade inserida ao processo.

Assim, a colaboração e criatividade têm assumido papel estratégico na promoção da inovação, junto ao design e à gestão da inovação. Em um processo de inovação dirigido pelo design (design driven innovation) projeta-se novos significados para a sociedade, para além da tecnologia.

Por fim, todas essas questões são convergentes em uma nova economia que se difunde na Sociedade Contemporânea do século 21, a Economia Criativa.

\section{Agradecimentos}

Nossos agradecimentos à Coordenação de Aperfeiçoamento de Pessoal de Nível Superior (CAPES) pelo apoio financeiro destinado à tese de doutorado vinculada a este trabalho. 


\section{Referências Bibliográficas}

BARAUNA, D.; SOUZA, S.; ZAMONER, M. T. D.C; RAZERA, D. L. Advanced Materials in Design for Innovation from the 21st Century: Context and Meaning. DAT Journal, v.2, n.2, p91-107, 2017. https://doi.org/10.29147/2526-1789.DAT.2017v2i2p91-107

BARAUNA, D.; RAZERA, D. L. Desenho de um novo mundo material: uma proposição de processos integrados e de aprendizagem. In: VI Encontro de Sustentabilidade em Projeto, ENSUS 2019, Florianópolis. Anais ENSUS. Florianópolis: UFSC/VIRTUHAB, v. 7. p. 255-266, 2019.

BAXTER, M. Projeto de produto: guia prático para o design de novos produtos. 3ed. São Paulo: Blucher, 2011.

BAYKARA, T. From the classical scheme to a smart/functional materials system: a generic transformation of advanced materials technologies. Procedia-Social and Behavioral Sciences, n.181, p. $79-88,2015$. https://doi.org/10.1016/j.sbspro.2015.04.868

BELL, B. Material intelligence: an overview of new materials for manufacturers. PFInnovation, Canadá, 2011.

CGEE. Materiais avançados no Brasil 2010-2022. Brasília: Centro de Gestão e Estudos Estratégicos, 2010.

CHEN, A. C. Exploring the relationship between technology diffusion and new material diffusion: the example of advanced ceramic powders. Technovation, v.32, p. 163 - 167, 2012. https://doi.org/10.1016/j.technovation.2011.10.008

DOBRZANSKI, L. A. Significance of materials science for the future development of societies. Journal of Materials Processing Technology, n. 175, p. 133-148, 2006. https://doi.org/10.1016/j.jmatprotec.2005.04.003

KULA, D.; TERNAUX, E. Materiologia: o guia criativo de materiais e tecnologias. São Paulo: Senac, 2012.

MAINE, E. M. A. Innovation and adoption of new materials (Doctoral thesis), 2000. https://doi.org/10.17863/CAM.14111

MAINE, E; PROBERTB, D.; ASHBY M. Investing in new materials: a tool for technology managers. Technovation, n.25, p.15-23, 2005. https://doi.org/10.1016/S0166-4972(03)00070-1

MCDOWELL, D. L.; STORY, T. L. New directions in materials design science and engineering (Mds\&E). 1998. In: Report of a Workshop Sponsored by the US National Science Foundation: Georgia Institute of Technology and Morehouse College, Atlanta, GA, 1998.

MEDINA, H. V. NAVEIRO, R. M. Materiais avançados: novos produtos e novos processos na indústria automobilística. Revista Produção, Belo Horizonte v.8, n.1, p. 29-44, 1998. http://dx.doi.org/10.1590/S0103-65131998000100003

MESSER, M.; PANCHAL, J. H.; ALLEN, J. K.; McDOWELL, D. L.; MISTREE, F. A function-based approach for integrated design of material and product concepts. In: ASME 2007 Design Engineering Technical Conferences, September 4-7, 2007, Las Vegas, Nevada, USA, 2007. pp. 799-827. https://doi.org/10.1115/DETC2007-35743

MORAES, D. Limites do Design. 2ed. São Paulo: Studio Nobel, 1999.

NASSOUR, A. C. Novos materiais: o homem cria um novo mundo. Revista Eletrônica de Ciências, v.44, p. 1-6, 2008. 
NATIONAL RESEARCH COUNCIL. Accelerating Technology Transition: Bridging the Valley of Death for Materials and Processes in Defense Systems. Washington: National Academies Press - NAP, 2004.

NAVARRO, R. F. A evolução dos materiais - Parte 1: da Pré-história ao início da Era Moderna Revista Eletrônica de Materiais e Processos. v.1, n.1, p. 01-11, 2006.

OLSON, G. B. Beyond discovery: design for a new material world. Calphad, v. 25, n. 2, p. 175 - 190, 2001. https://doi.org/10.1016/S0364-5916(01)00041-4

OLSON, G. B. Genomic materials design: the ferrous frontier. Acta Materialia, v. 61, n. 3, p. 771-781, 2013. https://doi.org/10.1016/j.actamat.2012.10.045

PADILHA, A. F. Materiais de engenharia: microestrutura e propriedades. Curitiba: Hemus, 2000.

THONG, C, ANDERSON, L, JACKSON, S. Design, science and engineering collaboration for developing new materials and technologies: a case study of outdoor café furniture. In: Cumulus 38 South Conference: Hemespheric Shifts Across Learning, Teaching and Research. November 12-14, 2009, Melbourne, Australia, 2009.

VERGHESE, G. Material change agents and their dangerous ideas. book: Interior Tactics, Interior Tools 2008.

VERGESHE, G. 20-20 Vision with the margin at the centre of design. In: Tenth Humanities Graduate Research Conference: Creative Margins, At Curtin University, Perth, Australia, 2009.

VOROS, A. L. S. A. Design e modos de vida: uma escuta para conexões entre objetos e valores contemporâneos. Dissertação (Mestrado em Design) Universidade Anhembi Morumbi, São Paulo, 2012. http://sitios.anhembi.br/tedesimplificado/handle/TEDE/1616

XIONG, H.; SUN, S.; JIANG, Y. Application of modern new materials in product design. In: ComputerAided Industrial Design and Conceptual Design, 2008. CAID/CD 2008. 9th International Conference on. 22-25 November 2008, Kunming, China: IEEE, v.9, p. 759-764, 2008. https://doi.org/10.1109/CAIDCD.2008.4730675

XXVI PRÊMIO JOVEM CIENTÍSTA. Aplicação e desenvolvimento de materiais esportivos. In: Kit Pedagógico. Caderno de Conteúdo, 2012. Disponível em: <http://estatico.cnpq.br/portal/premios/2013/pjc/imagens/publicacoes/07_KitXXVIPJC_CadernoCo nteudo_Cap3.pdf>. Acesso em: Jul. 2014.

ZAKON, A.; NASCIMENTO, J. L. N.; SZANJBERG, M. Algumas diferenças entre cientistas, engenheiros, técnicos e tecnólogos. Opinião. AdUFRJ/Seção Sindical, v. 11, 2003.

Debora Barauna dbarauna@unisinos.br

Dalton Luiz Razera daltonrazera@ufpr.br

Michele Tais D. C. Zamoner mizamoner@gmail.com

Denise Abatti Kasper Silva dabatti@univille.br 